\title{
Immunological detection of Bacteroides fragilis in clinical samples
}

\author{
SHEILA PATRICK, LINDA D. STEWART*, N. DAMANI*, K. G. WILSON, DEBORAH A. LUTTON, \\ M. J. LARKIN $\dagger$, I. POXTON $\ddagger$ and R. BROWN $\ddagger$
}

\begin{abstract}
Department of Microbiology and Immunobiology, School of Clinical Medicine, Queen's University of Belfast, Grosvenor Road, Belfast BT12 6BN, "Department of Microbiology, Craigavon Area Hospital, 68 Lurgan Road, Portadown, Co. Armagh BT63 50Q, † Division of Molecular Biology, School of Biology and Biochemistry, Queen's University of Belfast, Medical Biology Centre, Lisburn Road, Belfast BT7 INN and $\ddagger$ Department of Medical Microbiology, University of Edinburgh, Teviot Place, Edinburgh EH8 9AG
\end{abstract}

\begin{abstract}
Summary. A monospecific polyclonal antiserum, prepared against Bacteroides fragilis common polysaccharide antigen purified by polyacrylamide gel immunoblot detected $B$. fragilis, $B$. thetaiotaomicron, $B$. ovatus and Prevotella melaninogenica in pus samples from various anatomical sites by immunofluorescence microscopy of the pus. With standard clinical laboratory culture methods, $36 \%$ of 147 samples were positive for one or more of the above bacteria. Of these, $B$. fragilis accounted for $33 \%$. By immunofluorescent labelling of pus with the common antigen antiserum the detection of these bacteria in the samples increased to $50 \%$. All nine of the blood cultures in which $B$. fragilis was detected by culture contained bacteria positive for the common antigen. Immunofluorescent labelling of pus samples with a selection of monoclonal antibodies specific for surface polysaccharides which are known to be antigenically variable in culture in vitro and in an animal model of infection showed that these polysaccharides are also variable in natural infection. The results indicate that the common polysaccharide antigen, in contrast to the variable surface polysaccharides, is a suitable target for the immunodetection of $B$. fragilis in clinical samples from a range of anatomical sites.
\end{abstract}

\section{Introduction}

Bacteroides fragilis is the gram-negative obligately anaerobic bacterium most commonly isolated from clinical specimens. It has been isolated, either alone or as a component of mixed infection, from a range of sites in the body, including the peritoneal cavity, genito-urinary tract, blood, lungs and perianal area. ${ }^{1}$ The pathogenic contribution of $B$. fragilis in these mixed infections is apparent when the anaerobes are not taken into account in the treatment of the infection and this fails to resolve. ${ }^{2} B$. fragilis is a commensal of the gut and can account for up to $13 \%$ of the faecal flora whereas other members of the "fragilis group" of Bacteroides spp. (Bacteroides sensu stricto), such as B. vulgatus, predominate. However, in the adherent colonic mucosal flora, $B$. fragilis is the predominant Bacteroides spp. with an estimated incidence of c. $42 \%{ }^{3}$

As a result of difficulties in maintaining the viability of $B$. fragilis during the transportation of clinical specimens and the time taken to culture the bacteria, direct immunological detection of $B$. fragilis in clinical material by immunofluorescence microscopy has been studied by several workers. These studies have involved the use of a commercially available kit (Fluoretec, Pfizer Diagnostics) based on pooled rabbit polyclonal antisera specific for type strains of Bacteroides spp. (B. fragilis, B. vulgatus, B. distasonis, $B$. ovatus and $B$. thetaiotaomicron). The detection of Bacteroides spp. by immunofluorescence when compared with culture were reported as $81 \%,{ }^{4} 87 \%^{5}$ and $97 \% .{ }^{6}$ The usefulness of this kit as a monitor of faecal contamination of water has also been examined; however, it was not considered to be sufficiently sensitive for the routine monitoring of faecal contamination of disinfected drinking water. The potential diagnostic use of a mouse monoclonal antibody (MAb) specific for the core region of the lipopolysaccharide (LPS) has also been investigated. ${ }^{8}$ Although this MAb gave good specific labelling of $B$. fragilis and reacted with $96 \%$ of the clinical isolates examined, it labelled only c. $10 \%$ of the bacteria within a given strain. We have shown previously that 
labelling with MAbs specific for polysaccharide epitopes demonstrates within-strain antigenic variation in $B$. fragilis $^{9}$ and it appears that these variable epitopes are immunodominant. Polyclonal antisera raised to whole bacterial cells are also strain specific. This could explain the lack of agreement between the detection of $B$. fragilis by culture and immunofluorescence microscopy in these earlier studies. Therefore, there is a need to identify a non-variable antigen common to $B$. fragilis that could form the basis of an immunodiagnostic test.

In the present study, the suitability of the common polysaccharide antigen described by Poxton and Brown ${ }^{10}$ was investigated. This polysaccharide antigen migrates behind the rough form of the LPS and before the smooth form of the LPS on polyacrylamide gel electrophoresis and was reported to be common to seven strains of $B$. fragilis examined by immunoblotting with mono-specific polyclonal antiserum.

This study examined, by immunofluorescence microscopy, the reactivity of a monospecific polyclonal antiserum raised against this polysaccharide antigen with pus samples (and the corresponding pure culture isolates) from a range of different body sites and blood culture samples. The corresponding reactivity of a number of MAbs specific for variable polysaccharide antigens of $B$. fragilis was also examined.

\section{Materials and methods}

\section{Bacterial strains}

The strains used in this study were $B$. fragilis NCTC 9343 (National Collection of Type Cultures, Colindale Avenue, London), NCTC 10584, ATCC 23745 (American Type Culture Collection, Rockville, MD, USA); B. vulgatus NCTC $10583 ;$ B. thetaiotaomicron NCTC 10582; B. ovatus ATCC 8483; B. distasonis ATCC 8503; and clinical isolates of $B$. fragilis and other Bacteroides spp. obtained from Craigavon Area Hospital, Belfast City Hospital and Royal Victoria Hospital NI (designated LS, JC and including one metronidazole resistant strain, $\mathrm{BCH} 1 \mathrm{Mz}^{\mathrm{r}}$ ), the Free University, Amsterdam, NL (designated BE) and University of Edinburgh, Scotland (designated GNAB). The Escherichia coli and Staphylococcus aureus strains used were recent clinical isolates from the bacteriology laboratory at Craigavon Area Hospital, Northern Ireland.

\section{Specimens}

The clinical samples were all obtained in Northern Ireland. The majority came from Craigavon Area Hospital, Craigavon; however, a few were also obtained from South Tyrone Hospital, Tyrone, Daisy Hill Hospital, Newry and the Royal Victoria Hospital, Belfast.

Ninety-eight pus samples (Study 1) which had been sent to the laboratories for routine diagnostic testing were examined in detail and information concerning treatment was recorded from the patient's case history where available. These samples were subject to routine laboratory culture techniques (as detailed below) and examined by immunofluorescence microscopy for their reactivity with rabbit polyclonal antiserum to $B$. fragilis common polysaccharide antigen (CAg antiserum) and seven mouse MAbs specific for $\boldsymbol{B}$. fragilis as detailed below. The pure culture isolates of $B$. fragilis, other Bacteroides spp. and Prevotella melaninogenica obtained from these samples were also examined by immunofluorescence microscopy for their reactivity with the CAg antiserum and the seven MAbs. A further 49 pus samples (Study 2) were examined for the presence or absence of $B$. fragilis by routine diagnostic methods and their reactivity with the $\mathrm{CAg}$ antiserum by immunofluorescence microscopy.

Ten blood culture bottles of which nine were culture-positive for Bacteroides spp. were examined for their reactivity with the CAg antiserum and three of these were also examined with the MAbs.

The pus samples were obtained by surgical drainage or aspiration and were placed in sterile bottles in volumes of $1-20 \mathrm{ml}$. The pus samples were either plated out directly (as detailed below) or inoculated into Brain Heart Infusion Broth (Unipath) for $24 \mathrm{~h}$. Blood culture bottles (Roche Diagnostic System) were inoculated with $10 \mathrm{ml}$ of the patient's blood and incubated at $37^{\circ} \mathrm{C}$ aerobically and anaerobically for a total of 7 days. The cultures were examined by eye for bacterial growth three times a day for the first $48 \mathrm{~h}$ and once a day for the next 5 days. If growth was apparent in the anaerobic blood culture bottle, a sample was seeded to anaerobic blood agar and the identification procedures performed as detailed below.

\section{Identification by culture}

Samples were routinely plated on the following agar media: horse blood agar (BA; Unipath); anaerobic horse blood agar (ABA; Gibco); colistin-nalidixic acid agar (CNA; Unipath); anaerobic blood agar plus gentamicin $50 \mu \mathrm{g} / \mathrm{ml}(\mathrm{ABA}+\mathrm{GM})$. After inoculation, antibiotic disks containing penicillin (1 unit) and gentamicin $(10 \mu \mathrm{g})$ were applied to the BA and CNA plates and the plates were incubated at $37^{\circ} \mathrm{C}$ in an aerobic atmosphere with $\mathrm{CO}_{2} 10 \%$. Disks containing penicillin (1 unit) and metronidazole $(5 \mu \mathrm{g})$ were applied to the $A B A+G M$ plate and gentamicin $(10 \mu \mathrm{g})$, penicillin (1 unit) and metronidazole $(5 \mu \mathrm{g})$ to the ABA plate. Cultures were incubated at $37^{\circ} \mathrm{C}$ in an atmosphere of $\mathrm{H}_{2} 10 \%, \mathrm{~N}_{2} 80 \%$ and $\mathrm{CO}_{2} 10 \%$ in an anaerobic cabinet (Forma Scientific).

The aerobic plates were examined for colonies after 24 and $48 \mathrm{~h}$ and the isolates were gram-stained. Coliform isolates were identified with the API20E system (bioMérieux), S. aureus with Staphaurex (Murex Diagnostic), and Streptococcus spp. and Enterococcus spp. with the Lancefield serotyping kit Streptex (Murex Diagnostic). Streptococci which 
could not be classified with the Streptex kit were identified with the API20 STREP (bioMerieux). Small pin head colonies with a characteristic sweet smell that were identified as Group A, C or F, or were otherwise unidentifiable, were classified as Str. milleri.

The anaerobic plates were examined after at least $48 \mathrm{~h}$ and metronidazole-sensitive organisms were gram-stained. Single colonies were re-streaked on $\mathrm{ABA}$ and after $24 \mathrm{~h}$ the following tests were performed with the pure cultures: API20A (bioMérieux), ATB 32A (bioMérieux) and the Mastring ID 8 (Mast Laboratories).

Pure culture in brain heart infusion broth with glycerol $10 \%$, pus samples and blood cultures were stored in liquid nitrogen for future examination.

\section{Preparation of antiserum}

Monospecific polyclonal antiserum was raised to $B$. fragilis NCTC 9343 as described previously. Briefly, the monospecific polyclonal antiserum described by Poxton and Brown ${ }^{10}$ was used to identify the common polysaccharide antigen on guide strips of nitrocellulose after SDS-PAGE and immunoblotting. Small pieces of nitrocellulose containing the common antigen were cut from the unlabelled part of the nitrocellulose and dissolved in dimethylsulphoxide. This was mixed with an equal volume of Freund's complete adjuvant (Difco) and $0 \cdot 1-\mathrm{ml}$ amounts were inoculated subcutaneously at four sites on the back of a New Zealand White rabbit. Subsequently, the antigen in Freund's incomplete adjuvant was inoculated four times at $c$. 2week intervals. A further two inoculations of antigen in PBS were made at approximately monthly intervals and the rabbit was bled after each booster dose. Antisera were tested by both immunofluorescence microscopy and immunoblotting.

\section{Immunofluorescence microscopy}

Samples $(10 \mu 1)$ from blood culture bottles or pus were applied to one well of a multi-well slide (Flow Laboratories). Four doubling dilutions in phosphatebuffered saline (PBS) were made along the length of the slide. For pure bacterial cultures, a suspension of c. $10^{7}$ bacteria/ml in PBS $(30 \mu \mathrm{l})$ was applied to the slide. A duplicate series of dilutions of each sample was made on the same slide. The slides were air-dried and then fixed in methanol $100 \%$ for $10 \mathrm{~min}$ at $-20^{\circ} \mathrm{C}$. Slides with pus or blood culture samples were blocked with undiluted human serum for $10 \mathrm{~min}$. For single labelling, the slides were incubated with either undiluted murine MAb supernates or polyclonal rabbit antiserum diluted 1 in 100 in PBS for 45 min, washed in PBS and then incubated for $45 \mathrm{~min}$ with sheep antirabbit fluorescein conjugate (Sigma) diluted 1 in 100 in PBS. After a final wash, the slides were examined with a Leitz fluorescence microscope. For dual labelling, the slides were incubated with MAb supernates, washed, incubated with polyclonal rabbit antiserum, washed and incubated with sheep anti-rabbit fluorescein and goat anti-mouse rhodamine diluted 1 in 100 before a final wash. ${ }^{11}$ To estimate the sensitivity of the labelling, Evan's Blue (Gurr; $0.05 \%$ w/v) was included during incubation with the conjugate. The slides were mounted with glycerol PBS containing an anti-photobleaching agent (Citifluor; Agar Scientific Ltd, Essex). The proportion of bacteria labelled with the antisera was determined by eye and confirmed by photographing the same field of view with filters suitable for viewing the green fluorescence of the fluorescein followed by filters suitable for viewing the red fluorescence of the Evan's blue staining with a fluorescence microscope. The percentage of bacteria labelled with the antisera was estimated by counting the total number of bacteria in each of the photographs. A minimum of 200 bacteria were counted for each estimation. Similarly, where samples were doubly labelled with a MAb and polyclonal antiserum, fields of view were photographed with filters suitable for viewing either the fluorescein (green) or rhodamine (red) dyes. Double-colour photographic exposure of the same field with the two different filter types sequentially were also examined.

Clinical samples were scored positive by immunofluorescence if at least three positive bacterial cells were observed in a sample after extensive examination of the micro-well slide. Each slide was examined for c. $10 \mathrm{~min}$. The intensity of fluorescence was also noted.

\section{Results}

\section{Sensitivity of labelling with the polyclonal antiserum to $C A g$}

A number of different strains of $B$. fragilis and related bacteria were examined for their reactivity with rabbit polyclonal anti-serum specific for $\mathrm{CAg}$ by immunofluorescence microscopy. The total number of bacteria was estimated microscopically after being stained with Evan's blue. All the strains of $B$. fragilis examined were positive for $\mathrm{CAg}$ and between 80 and $100 \%$ of the bacteria were labelled with the polyclonal antiserum (table I). B. ovatus and B. thetaiotaomicron

Table I. Sensitivity of labelling of $B$. fragilis strains with the antiserum to the $\mathrm{CAg}$

\begin{tabular}{lclc}
\hline Strain no. & $\begin{array}{c}\text { Bacteria } \\
\text { positive (\%) }\end{array}$ & Strain no. & $\begin{array}{c}\text { Bacteria } \\
\text { positive (\%) }\end{array}$ \\
\hline NCTC 9343 & & LS54* & 99 \\
EDL population & 103 & LS66* & 98 \\
SC population & 95 & LS67* & 97 \\
LC population & 92 & BE1 & 80 \\
NCTC 10584 & 99 & BE3 & 89 \\
ATCC 23745 & 96 & JC6 & 98 \\
GNAB 4 & 86 & JC15 & 100 \\
GNAB 82 & 96 & JC17 & 98 \\
GNAB 92 & 98 & JC19 & 100 \\
BCH1 Mz & 100 & &
\end{tabular}

EDL, electron dense layer; SC, small capsule; LC, large capsule. *Clinical isolates from the current study. 
Table II. Detection of Bacteroides spp. and related genera in pus samples by culture and immunolabelling with antiserum to CAg (Study 1)

\begin{tabular}{|c|c|c|c|c|}
\hline \multirow[b]{2}{*}{ Source of specimen } & \multirow{2}{*}{$\begin{array}{l}\text { Number of } \\
\text { samples } \\
\text { received }\end{array}$} & \multicolumn{2}{|c|}{ Number culture-positive for } & \multirow{2}{*}{$\begin{array}{c}\text { CAg positive, } \\
\text { Bacteroides } \\
\text { and Prevotella } \\
\text { culture- } \\
\text { negative }\end{array}$} \\
\hline & & B. fragilis* & $\begin{array}{c}\text { Other } \\
\text { Bacteroides and } \\
\text { Prevotella } \mathrm{spp} .\end{array}$ & \\
\hline Perianal abscess & 15 & 11 & 0 & 2 \\
\hline Abdominal abscess & 10 & 4 & 1 & 2 \\
\hline Pilonidal abscess & 9 & 2 & 5 & 1 \\
\hline Bartholin's abscess & 6 & 1 & 0 & 0 \\
\hline Ischiorectal abscess & 4 & 2 & 1 & 1 \\
\hline Vaginal abscess & 1 & 1 & 0 & 0 \\
\hline Diverticular abscess & 1 & 1 & 0 & 0 \\
\hline Groin abscess & 3 & 1 & 0 & 2 \\
\hline Groin neoplasm abscess & 1 & 1 & 0 & 0 \\
\hline Colostomy & 1 & 1 & 0 & 0 \\
\hline Abscess at pacemaker & 1 & 0 & 0 & 0 \\
\hline Haematoma & 1 & 0 & 0 & 0 \\
\hline Skin wounds & 31 & 0 & 0 & 2 \\
\hline Pleural fluid & 2 & 0 & 0 & 1 \\
\hline Gall bladder abscess & 2 & 0 & 0 & 1 \\
\hline Liver abscess & 3 & 0 & 0 & 0 \\
\hline Brain abscess & 2 & 0 & 0 & 0 \\
\hline Pancreatic abscess & 1 & 0 & 0 & 0 \\
\hline Peritoneal fluid & 1 & 0 & 0 & 0 \\
\hline Parotid abscess & 1 & 0 & 0 & 0 \\
\hline Subphrenic abscess & 2 & 0 & 0 & 0 \\
\hline Total & 98 & 25 & 7 & 12 \\
\hline
\end{tabular}

*All these samples were positive when directly labelled with the CAg antiserum.

Table IIIA. Bacteria isolated from $B$. fragilis culture-positive pus samples
Organisms isolated

Abscess site

B. fragilis

B. fragilis, E. coll

B. fragilis, Str. milleri

B. fragilis, Str. milleri, E. coli

B. fragilis, Str. agalactiae

B. fragilis, Str. viridans

B. fragilis, E. coli, Str. milleri,

Str. agalactiae

B. fragilis, E. coli, Str. agalactiae Diverticular (1), groin neoplasm (1)

B. fragilis, Group C streptococci Perianal (1)

B. fragilis, $P$. melaninogenica Abdominal (1)
Perianal (2), colostomy (1)

Perianal (2), ischiorectal (1)

Abdominal (1), perianal (2)

groin (1), pilonidal (1)

Perianal (3), abdominal (1)

iginal (1), ischiorectal (1)

Abdominal (1)

Bartholin's (1) were labelled with lower intensity but similar sensitivity to $B$. fragilis, and $B$. distasonis gave negative results.

\section{Detection of Bacteroides spp. in pus samples}

The source of the 98 pus samples examined in Study 1, the culture results for Bacteroides spp. and related genera and the reactivity with the CAg antiserum by immunofluorescence microscopy are shown in table II. The bacteria isolated from samples positive for $B$. fragilis, Bacteroides spp. and the related genus Prevotella by initial culture are shown in tables IIIA and IIIB respectively. Pus samples that were culturenegative for Bacteroides and Prevotella spp., but
Table IIIB. Bacteria isolated from Bacteroides spp. and Prevotella spp. culture-positive pus samples

\begin{tabular}{ll}
\hline Organisms isolated & \multicolumn{1}{c}{ Abscess site } \\
\hline $\begin{array}{l}\text { P. melaninogenica } \\
\text { P. melaninogenica, }\end{array}$ & $\begin{array}{l}\text { Abdominal wound (1) } \\
\text { Peptostreptococcus sp. }\end{array}$ \\
$\begin{array}{l}\text { P. melaninogenica, } \text { E. coli, Str. milleri } \\
\text { B. thetaiotaomicron, } \text { E. coli, Str. milleri, },\end{array}$ & Pilonidal (2) \\
Proteus mirabilis & \\
Bacteroides sp., Str. milleri & \\
Bacteroides sp., Str. milleri, & Pilonidal (1) \\
Peptostreptococcus sp. & Pilonidal (1) \\
\end{tabular}

Table IV. Bacteria isolated on repeat culture from CAgpositive but Bacteroides and Prevotella culture-negative pus samples

Organisms initially isolated

Abscess site

\begin{tabular}{|c|c|}
\hline None & $\begin{array}{l}\text { Buttock (skin wound)*†, labial (skin } \\
\text { wound), abdominal } \dagger\end{array}$ \\
\hline E. coli & $\begin{array}{l}\text { Gall bladder, pilonidal } \dagger \text {, groin, } \\
\text { perianal, abdominal }{ }^{+}+\end{array}$ \\
\hline Str. milleri & Pleural fluid, ischiorectal*† \\
\hline E. coli, Str. milleri & Perianal* \\
\hline S. aureus, Str. pyogenes & Groint \\
\hline
\end{tabular}

* $B$. fragilis isolated after re-culture of the sample.

+ Positive with one or more $B$. fragilis-specific MAb (see table IX)

positive by immunofluorescence with the $\mathrm{CAg}$ antiserum are detailed in table IV. These 12 specimens which were negative for Bacteroides and Prevotella 
Table V. Detection of $B$. fragilis in pus samples by both culture and immunolabelling with antiserum to the CAg. (Study 2)

\begin{tabular}{cccc}
\hline Source of specimen & $\begin{array}{c}\text { Number of } \\
\text { samples } \\
\text { received }\end{array}$ & $\begin{array}{c}\text { Number } \\
\text { culture- } \\
\text { positive for } \\
\text { B. fragilis* }\end{array}$ & $\begin{array}{c}\text { CAg-positive, } \\
\text { Bacteroides } \\
\text { culture- } \\
\text { negative }\end{array}$ \\
\hline
\end{tabular}

Perianal abscess

Pilonidal abscess

Unspecified pus

Abdominal abscess

Scrotum abscess

Pelvic abscess

Mastoid maxillary sinus

Suprapubic abscess

Pleural cavity

Hernia wound abscess

Pouch of Douglas

Neck abscess

Quinsy

Wound abscess

Abdominal drain fluid

Tibial abscess

Breast abscess

Back abscess

Gall bladder abscess

Total

\begin{tabular}{rrr}
9 & 9 & 0 \\
8 & 4 & 2 \\
7 & 3 & 1 \\
4 & 2 & 1 \\
4 & 1 & 2 \\
2 & 0 & 2 \\
3 & 0 & 2 \\
1 & 1 & 0 \\
1 & 1 & 0 \\
1 & 0 & 1 \\
1 & 0 & 0 \\
1 & 0 & 0 \\
1 & 0 & 0 \\
1 & 0 & 0 \\
1 & 0 & 0 \\
1 & 0 & 0 \\
1 & 0 & 0 \\
1 & 0 & 0 \\
1 & 0 & 0 \\
49 & 21 & 11 \\
\hline
\end{tabular}

*All except one of these samples were also positive when labelled with the CAg antiserum.

spp. on initial routine testing in the laboratory, but immunofluorescence-positive with the CAg antiserum, were retrieved from storage in liquid nitrogen and re-cultured. Of these, $B$. fragilis was isolated from four (table IV).
Of a further 49 pus samples examined in Study 2, 21 were positive by culture for $B$. fragilis. Eleven of the culture-negative samples were positive by immunofluorescence with CAg antiserum (table V). Immunofluorescence labelling of a typical pus sample is illustrated in fig. 1. With the exception of one sample in Study 2, if a sample was positive for $B$. fragilis on culture it was also positive by immunofluorescence with the CAg antiserum. Of the samples positive for Bacteroides spp. or $P$. melaninogenica, or both, only one (no. 3), which was positive for an unidentified Bacteroides sp., was negative by immunofluorescence.

In total, $36 \%$ of the pus samples were positive for $B$. fragilis and related bacteria by culture and $50 \%$ were positive by immunofluorescence microscopy with the CAg antiserum.

\section{Investigation of the patients' case histories}

The antibiotic treatment recorded in the case histories of patients whose specimens were either CAg antiserum- or culture-positive for $\boldsymbol{B}$. fragilis is given in table VI. Although metronidazole was prescribed before drainage of the abscesses, samples nos. 11 and 15 were still positive for $B$. fragilis on culture. One sample (no. 68) was culture-negative and the antibiotic treatment was changed to co-fluampicil; however, the current study indicated that this sample was CAgpositive. Examination of the case histories also revealed that four patients had recurrent infections at the same anatomical site (table VII). 
Table VI. Antibiotics* administered to patients with either $B$. fragilis and related bacteria culture- and CAg-positive (A) or only CAg-positive (B) pus samples. (Study 1)

\begin{tabular}{|c|c|c|}
\hline \multirow{2}{*}{ Sample no. } & \multicolumn{2}{|c|}{ Antibiotic treatment } \\
\hline & before drainage & after drainage \\
\hline \multicolumn{3}{|l|}{ A } \\
\hline 4 & Co-fluampicil & NI \\
\hline 11 & Cefuroxime and metronidazole & Cefuroxime and metronidazole \\
\hline 14 & Co-fluampicil & NI \\
\hline 15 & Gentamicin and metronidazole & Gentamicin and metronidazole \\
\hline 18 & NI & Ampicillin and metronidazole \\
\hline 23 & Co-fluampicil & NI \\
\hline 56 & Flucloxacillin & NI \\
\hline 66 & Metronidazole and cefuroxime & Metronidazole and cefuroxime \\
\hline 73 & NI & Erythromycin \\
\hline 75 & Amoxycillin & NI \\
\hline 91 & Flucloxacillin & NI \\
\hline 96 & Co-fluampicil & NI \\
\hline \multicolumn{3}{|l|}{ B } \\
\hline 28 & NI & Gentamicin \\
\hline 68 & Co-amoxiclav and metronidazole & Co-fluampicil \\
\hline 70 & Co-fluampicil & NI \\
\hline 71 & Co-fluampicil & NI \\
\hline
\end{tabular}

NI, not indicated.

*Information obtained from patients' case histories, where available.

Table VII. Bacteria isolated from successive samples obtained from the same patient* based on information in patients' case histories

\begin{tabular}{|c|c|c|c|}
\hline Sample no. & Abscess site & Date & Culture result \\
\hline 23 & Ischiorectal & $\begin{array}{l}14.02 .89 \\
12.08 .90\end{array}$ & $\begin{array}{l}{ }^{*} \text { B. fragilis, Str. milleri } \\
\text { B. fragilis, Str. milleri }\end{array}$ \\
\hline 70 & Pilonidal & $\begin{array}{l}29.11 .89 \\
28.05 .90\end{array}$ & $\begin{array}{l}\text { *Str. milleri } \\
\text { Str. milleri, B. fragilis }\end{array}$ \\
\hline 80 & Ischiorectal/perianal & $\begin{array}{l}14.07 .89 \\
04.04 .90 \\
\\
30.04 .90 \\
24.08 .90 \\
12.03 .91\end{array}$ & $\begin{array}{l}\text { No growth } \\
\text { Str. milleri, B. fragilis, } \\
P . \text { melaninogenica } \\
\text { *Str. milleri, B. fragilis } \\
\text { No growth } \\
\text { Str. milleri, B. fragilis, } \\
\text { P. melaninogenica }\end{array}$ \\
\hline 95 & Ischiorectal/perianal & $\begin{array}{l}29.09 .90 \\
23.12 .87\end{array}$ & $\begin{array}{l}{ }^{*} \text { Str. milleri, B. fragilis, E. coli } \\
\text { Str. milleri, B. fragilis, E. coli }\end{array}$ \\
\hline
\end{tabular}

*Denotes pus sample examined in Study 1.

\section{Detection of $B$. fragilis from blood culture bottles}

Nine blood culture bottles from which $B$. fragilis was isolated, and one from which $B$. distasonis was isolated, were examined for their reactivity with the $\mathrm{CAg}$ antiserum. All nine cultures positive for $B$. fragilis were also positive by immunofluorescence with the CAg antiserum; however, the $B$. distasonis-positive blood culture bottle was CAg-negative. Fig. 2 illustrates typical labelling of a blood culture sample.

\section{Reactivity of $B$. fragilis clinical samples and pure} cultures with murine MAbs

Twenty-five pus samples (from Study 1) and three blood culture bottles, which were all $\mathrm{CAg}$ positive, were examined by immunofluorescence microscopy for their reactivity with six murine MAbs specific for the surface polysaccharides of $B$. fragilis associated with the large capsule and electron-dense layer populations. The corresponding pure culture isolated was also examined in each case. MAb QUBf5 is specific for the polysaccharide which forms on PAGE and immunoblotting a pattern similar to that of the $\mathrm{O}$-antigen of other bacterial species. The other MAbs are specific for higher molecular mass polysaccharide associated with the large capsule and electron-dense layer populations of $B$. fragilis. $^{9}$

Four of the pus samples did not react with any of the MAbs when examined directly and the rest reacted with a variable selection of the MAbs. All of the pure culture isolates of $B$. fragilis obtained from these samples were positive for at least one of the MAbs 




Fig. 2. Micrograph of blood culture labelled with rabbit antiserum to $\mathrm{CAg}$ and anti-rabbit immunoglobulin fluorescein conjugate $(\times 100)$.

(table VIII). There was no apparent relationship between the site of origin of the sample and the MAb labelling. Double labelling with both the CAg antiserum and the MAbs confirmed that the MAbs label a varying proportion of the $B$. fragilis cells present within a pure culture isolate. This was also the case when pus and blood culture samples were examined directly, without prior culture. Fig. 3 illustrates double labelling of a blood culture. MAb QUBf11, which reacted with all of the pure cultures and 24 of the 28 samples, labelled only $7-15 \%$ of the bacterial population. Labelling also indicated that outer membrane vesicles were present in the pus samples.

Examination of the samples that were culture negative for $B$. fragilis and related bacteria, but were positive with the CAg antiserum, indicated that seven of 12 of these samples also reacted with at least one of the MAbs (table IX). Samples from which Bacteroides spp. (other than B. fragilis) or P. melaninogenica, or both, were cultured and which were positive with the CAg antiserum (table IIIB), were negative with all the MAbs. This suggests that the polysaccharides recognised by these MAbs are specific to $B$. fragilis.

Sixteen of the pure culture isolates from Study 1 were examined for their reactivity with a MAb (Bf4) specific for a high molecular mass polysaccharide associated with the small capsule population of $B$. fragilis. ${ }^{12}$ An estimated $1 \%$, or less, of the bacteria within these populations were labelled with the MAb. Similarly, the proportion of the bacteria positive with the CAg antiserum, which were also positive with $\mathrm{MAb} \mathrm{Bf} 4$ when pus samples were examined directly, was $\leqslant 1 \%$.

\section{Discussion}

The results confirm that the heterogeneous labelling pattern of MAbs specific for high molecular mass polysaccharides of $B$. fragilis, previously observed in culture collection strains and in bacteria grown in a mouse model of peritoneal infection, ${ }^{13}$ also occurred in the recent clinical isolates of $B$. fragilis obtained in the study. Variable labelling was also apparent when pus samples were examined directly, without prior culture. Polysaccharides associated with the large capsule, small capsule and electron-dense layer populations ${ }^{\mathbf{9 , 1 2}}$ were all detected in the pus samples with the MAbs. This indicates that antigenic variation in the polysaccharides of $B$. fragilis is apparent during the course of natural infection and raises the possibility that antigenic variation of surface polysaccharides is related to the virulence of $B$. fragilis. Interestingly, there was no obvious relationship between the labelling pattern of the MAbs and a particular site of infection. It is possible that variable antigens are the immunodominant antigens in $B$. fragilis as inoculation with whole cells produces antisera that do not label all the bacterial cells in other strains ${ }^{\mathbf{1 4 , 1 5}}$ and are thus too insensitive for use as a diagnostic test for the presence of $B$. fragilis. Furthermore, all the polysaccharidespecific MAbs that were raised with whole bacteria as an inoculum were specific for the antigenically variable polysaccharides which are not expressed by all the bacteria within a given population of $B$. fragilis. ${ }^{16-18}$ It is possible that these immunodominant variable polysaccharides mask the common polysaccharide. The unsuitability of the variable polysaccharides as targets 
Table VIII. Reactivity of clinical samples (S) and the corresponding pure culture isolate $(C)$ of $B$. fragilis with MAbs specific for $B$. fragilis NCTC 9343 surface polysaccharides

\begin{tabular}{|c|c|c|c|c|c|c|c|c|c|c|c|c|}
\hline \multirow{3}{*}{$\begin{array}{l}\text { Site and } \\
\text { sample no. }\end{array}$} & \multicolumn{12}{|c|}{ Reaction with MAb QUBf } \\
\hline & \multicolumn{2}{|c|}{5} & \multicolumn{2}{|c|}{7} & \multicolumn{2}{|c|}{8} & \multicolumn{2}{|c|}{9} & \multicolumn{2}{|c|}{10} & \multicolumn{2}{|c|}{11} \\
\hline & $\mathrm{C}$ & $\mathrm{S}$ & $\mathrm{C}$ & $\mathrm{S}$ & $\mathrm{C}$ & $\mathrm{S}$ & $\mathrm{C}$ & $\mathrm{S}$ & $\mathrm{C}$ & $\mathrm{S}$ & $\mathrm{C}$ & $\mathrm{S}$ \\
\hline \multicolumn{13}{|c|}{ Perianal/ischiorectal abscess } \\
\hline 14 & + & + & + & + & - & - & + & - & + & + & + & + \\
\hline 16 & - & - & - & - & - & - & + & - & + & - & + & - \\
\hline 23 & - & - & - & - & - & - & - & - & - & - & $L$ & + \\
\hline 26 & - & - & - & - & - & - & - & - & + & - & + & - \\
\hline 52 & - & - & - & - & + & + & - & - & - & - & + & + \\
\hline 54 & + & + & + & + & + & + & - & - & + & - & + & + \\
\hline 61 & - & - & - & - & + & + & - & - & + & + & + & + \\
\hline 67 & + & + & + & + & + & + & - & - & - & - & + & + \\
\hline 75 & - & - & + & + & + & + & - & - & - & - & + & + \\
\hline 80 & + & + & + & + & + & + & + & + & + & + & + & + \\
\hline 87 & - & - & - & - & + & + & - & - & + & + & + & + \\
\hline 92 & + & + & - & - & + & + & - & - & + & + & + & + \\
\hline 95 & + & + & - & - & + & + & - & - & + & + & + & + \\
\hline \multicolumn{13}{|c|}{ Abdominal abscess } \\
\hline 11 & - & - & - & - & - & - & + & - & + & - & + & + \\
\hline 13 & - & - & - & - & - & - & - & - & - & - & + & - \\
\hline 66 & + & + & + & - & - & - & + & + & + & - & + & + \\
\hline 89 & - & - & + & + & + & + & - & - & - & - & + & + \\
\hline \multicolumn{13}{|c|}{ Pilonidal abscess } \\
\hline 56 & + & + & - & - & - & - & - & - & + & + & + & + \\
\hline 91 & - & - & - & - & + & + & - & - & + & + & + & + \\
\hline \multicolumn{13}{|c|}{ Vaginal/Bartholin`s abscess } \\
\hline 18 & + & + & + & + & + & + & - & - & - & - & + & + \\
\hline 76 & - & - & - & - & - & - & - & - & + & + & + & + \\
\hline \multicolumn{13}{|c|}{ Diverticular abscess } \\
\hline 84 & + & + & - & - & - & - & + & + & + & + & + & + \\
\hline \multicolumn{13}{|l|}{ Groin abscess } \\
\hline 73 & - & - & - & - & + & + & - & - & + & + & + & + \\
\hline \multicolumn{13}{|l|}{ Colostomy } \\
\hline 15 & - & - & - & - & - & - & - & - & - & - & + & - \\
\hline \multicolumn{13}{|c|}{ Groin neoplasm } \\
\hline 27 & + & + & - & - & + & + & + & + & + & + & + & + \\
\hline \multicolumn{13}{|l|}{ Blood culture } \\
\hline 39 & + & + & + & + & + & + & - & - & - & - & + & + \\
\hline 55 & + & - & - & - & + & + & - & - & + & + & + & + \\
\hline 98 & - & - & - & - & + & + & - & - & - & - & + & + \\
\hline
\end{tabular}

C. pure culture: S. pus sample.

for the immunological detection of $B$. fragilis in clinical samples is emphasised. The sensitivity of labelling is not sufficient to allow detection of $B$. fragilis in all instances. It is unlikely that even a mixture of these MAbs could be used effectively as some samples and isolates reacted with only one MAb.

In contrast, a monospecific polyclonal antiserum specific for the CAg of $B$. fragilis ${ }^{10}$ labelled between 80 and $100 \%$ of the bacteria within 16 strains of $B$. fragilis obtained from culture collections and isolates from Northern Ireland, Edinburgh and Amsterdam, and also populations of strain NCTC 9343 enriched for the large capsule, small capsule or electron-dense layer. The antiserum recognised $B$. fragilis, $B$. thetaiotaomicron, $B$. ovatus and $P$. melaninogenica but not $B$. vulgatus or $B$. distasonis. Why this polysaccharide is common to some species of Bacteroides and Prevotella but not other species of Bacteroides is unclear. Interestingly, there is evidence for the horizontal transfer of genes between Bacteroides and Prevotella spp. ${ }^{19}$ By labelling bacteria directly in pus samples, the detection of these species was increased above that obtained by culture only. The culturenegative immunofluorescence microscopy-positive samples are unlikely to represent false positives, as (i) four of these yielded viable $B$. fragilis when the isolation procedures were performed a second time from the stored pus samples, with particular care to minimise the exposure of the material to air and not as part of the normal day-to-day activity of the diagnostic laboratory, and (ii) a number of culture-negative samples were also positive by immunofluorescence microscopy with MAbs specific for $B$ : fragilis polysaccharides (table IX) which, because of the specificity of the MAbs, indicates that $B$. fragilis cells were present in these specimens but were non-viable. Therefore, this 

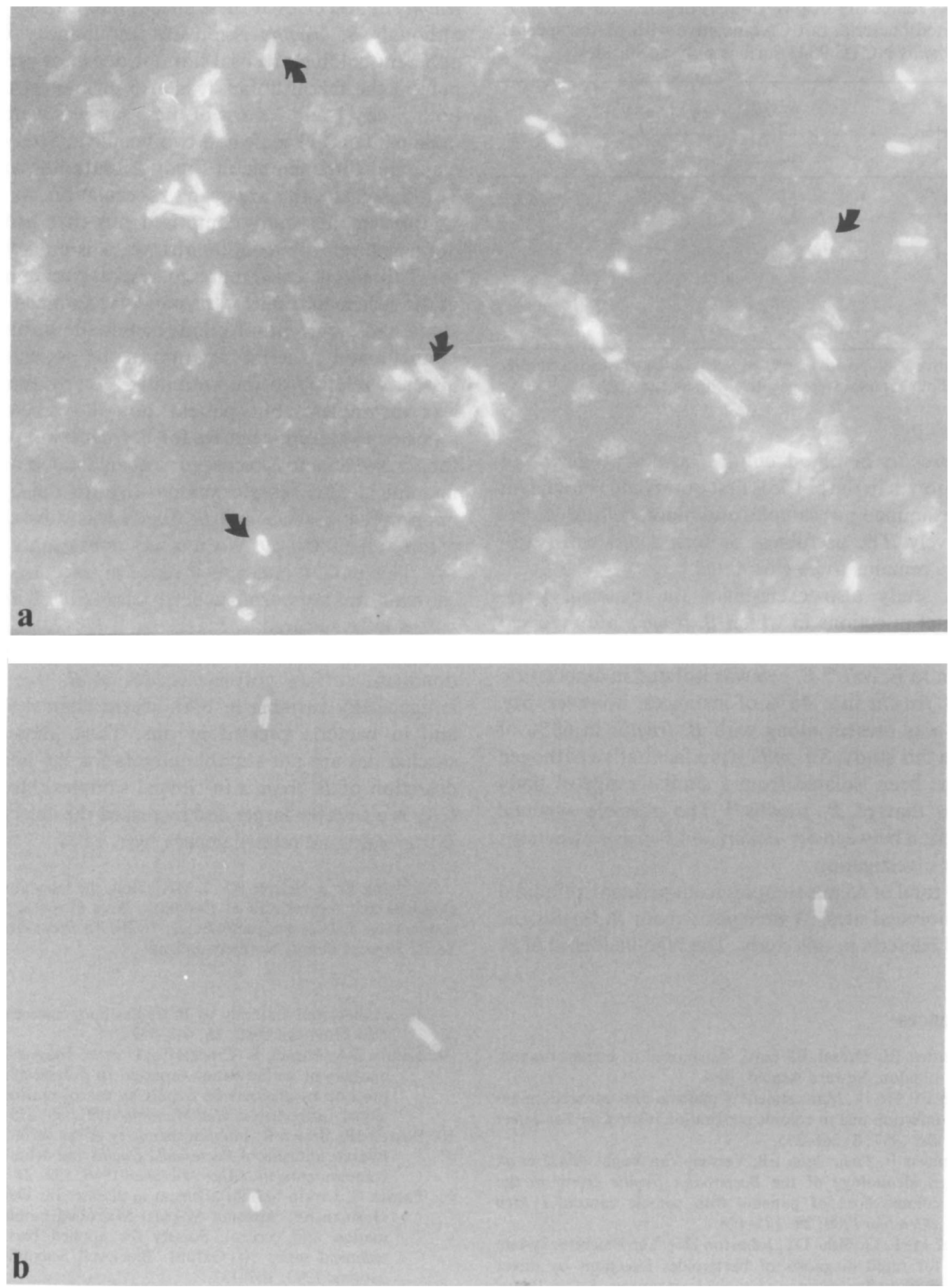

Fig. 3. Micrographs of blood culture labelled with (i) rabbit antiserum to $\mathrm{CAg}$ and anti-rabbit immunoglobulin fluorescein conjugate and (ii) mouse MAb QUBf11 and anti-mouse immunoglobulin rhodamine conjugate. The same field of view was photographed with filters to show: a, the green fluorescence of the fluorescein; $b$, the red fluorescence of the rhodamine $(\times 100)$.

study highlights the problems of detecting obligate anaerobes in clinical specimens by culture alone in a routine diagnostic laboratory and indicates that the $\mathrm{CAg}$ is a suitable target for immuno-detection. If the $\mathrm{CAg}$ antiserum alone had been used for the detection of $B$. fragilis and related bacteria, two samples that were positive by culture would have been mis-reported as negative. This represents an underestimate of $1-2 \%$ of the total of 147 samples examined in the study. In contrast with the current diagnostic laboratory culture methods, the incidence of $B$. fragilis and related bacteria was underestimated by $c .14 \%$. Although relatively few blood culture bottles were examined, in all nine instances where $B$. fragilis was isolated, the blood culture was positive with the CAg antiserum. Thus, there is the potential for immediate confirmation 
Table IX. Reactivity of pus samples negative for $B$. fragilis and related bacteria, but $\mathrm{CAg}$-positive with MAbs specific for $B$. fragilis NCTC 9343 surface polysaccharides

\begin{tabular}{lcccccc}
\hline & \multicolumn{7}{c}{ Reaction with MAb QUBf } \\
\cline { 2 - 7 } Sample no. & 5 & 7 & 8 & 9 & 10 & 11 \\
\hline 28 & - & + & - & + & - & + \\
$34^{*}$ & - & + & + & - & - & + \\
35 & - & + & - & - & - & + \\
$57^{*}$ & - & - & + & + & + & + \\
68 & - & - & + & - & + & + \\
$70^{*}$ & - & - & + & - & + & + \\
94 & + & - & + & - & + & +
\end{tabular}

- negative; + , positive. ${ }^{*} B$. fragilis culture positive after reculture. Four samples were negative with all the MAbs.

of $B$. fragilis in blood culture bottles at the point when growth in the bottle is first observed. The current study examined pus samples and blood culture bottles exclusively. The usefulness of such a test with swab samples remains to be elucidated.

This study also exemplifies the classical polymicrobial infections in which $B$. fragilis are involved and which have been much studied, in particular in relation to $E$. coli. ${ }^{20} E$. coli was isolated in association with B. fragilis in c. $46 \%$ of instances; however, Str. milleri was present along with $B$. fragilis in $68 \%$ of cases in this study. Str. milleri is a facultative pathogen that has been isolated from a similar range of body sites to that of $B$. fragilis. ${ }^{21}$ The possible synergic interaction between Str. milleri and $B$. fragilis warrants further investigation.

Of a total of 45 pus samples from perianal, pilonidal or ischiorectal sites, 39 were positive for $\boldsymbol{B}$. fragilis and related bacteria in this study. The high incidence of $B$.

\section{References}

1. Duerden BI, Drasar BS (eds). Anaerobes in human disease. London, Edward Arnold. 1991.

2. Tally FP. Ho JL. Management of patients with intraabdominal infection due to colonic perforation. Curr Clin Top Infect Dis $1987 ; 8$ : 266-295.

3. Namavar F. Theunissen EB, Verweij-Van Vught AMJJ et al. Epidemiology of the Bacteroides fragilis group in the colonic flora of patients with colonic cancer. $J$ Med Microbiol 1989; 29: 171-176.

4. Slack MPE, Griffiths DT, Johnston HH. The Fluoretec system for rapid diagnosis of bacteroides infections by direct immunofluorescence of clinical specimens. J Clin Pathol $1981: 34: 1381-1384$

5. Labbé M. Delamare N, Pepersack F, Crokaert F, Yourassowsky E. Detection of Bacteroides fragilis and Bacteroides melaninogenicus by direct immunofluorescence. J Clin Pathol 1980; 33: 1189-1192.

6. Holst E. Oscarson J, Mårdh P-A. Evaluation of two fluorescent test kits for detection of selected Bacteroides species in clinical specimens. Curr Microbiol 1979; 3: 133-136

7. Fiksdal L. Berg JD. Evaluation of a fluorescent antibody technique for the rapid enumeration of Bacteroides fragilis group of organisms in water. J Appl Bacteriol 1987: 62: $377-383$

8. Viljanen MK, Linko L, Lehtonen O-P. Detection of Bacteroides fragilis. Bacteroides thetaiotaomicron and Bacteroides oratus in clinical specimens by immunoffuorescence with a fragilis in abscesses at these sites is interesting in that, although $B$. fragilis is present in quantity in the adherent colonic mucosal flora, it does not predominate in the faecal flora. ${ }^{3}$ It is also of interest that in both Study 1 and 2 most of these samples were from male patients (11 male and two female in Study 1; 10 male and three female in Study 2; data not shown). The reasons for this are open to speculation. As all the samples in this study comprised pus that had been drained surgically from an abscess, it is unlikely that the $B$. fragilis detected represents faecal contamination of the specimen. Four of the patients examined in this study had recurrent infections at this site (table VII). Unfortunately, the information in the patients' case histories relating to the antibiotic therapy employed was incomplete, but patient no. 70 was initially reported as culture-negative for $B$. fragilis but positive for Str. milleri and is recorded as being treated with cofluampicil. This sample was positive for the CAg by immunofluorescence and $B$. fragilis was subsequently cultured from the pus when it was re-examined (table IV). This patient had a recurrence of infection at this site 6 months later, from which both B. fragilis and Str. milleri were cultured.

In conclusion, this study confirms that the immunodominant surface polysaccharides of $B$. fragilis are antigenically variable in both recent clinical isolates and in bacteria present in pus. Thus, these polysaccharides are not suitable targets for the immunodetection of $B$. fragilis in clinical samples. However, $\mathrm{CAg}$ is a suitable target and increased the detection of Bacteroides and related genera by c. $14 \%$.

We thank Dr S. Nelson, Mr J. McCallum, the laboratory staff, clinicians and theatre staff of Craigavon Area Hospital for their cooperation. L.D.S. was partly funded by the Southern Health and Social Services Board, Northern Ireland.

monoclonal antibody to $B$. fragilis lipopolysaccharide. $J$ Clin Microbiol 1988; 26: 448-452.

9. Lutton DA, Patrick S, Crockard AD et al. Flow cytometric analysis of within-strain variation in polysaccharide expression by Bacteroides fragilis by use of murine monoclonal antibodies. J Med Microbiol 1991; 35: 229-237.

10. Poxton IR, Brown R. Immunochemistry of the surface carbohydrate antigens of Bacteroides fragilis and definition of a common antigen. J Gen Microbiol 1986; 132: 2475-2481.

11. Patrick S, Larkin MJ. Attachment in disease. In: Denyer SP, Gorman SP, Sussman M (eds) Microbial biofilms: formation and control. Society for applied bacteriology technical series 30. Oxford: Blackwell Scientific Publications. 1993: 109-131.

12. Reid JH, Patrick S, Tabaqchali S. Immunochemical characterization of a polysaccharide antigen of Bacteroides fragilis with an IgM monoclonal antibody. J Gen Microbiol 1987 ; 133: $171-179$.

13. Patrick S. The virulence of Bacteroides fragilis. Rev Med Microbiol 1993; 4: 40-49.

14. Schwan A, Danielsson D. Forsum U. Demonstration of heatlabile antigen(s) of Bacteroides fragilis associated with nonhomogeneous immunofluorescent staining. Acta Pathol Microbiol Immunol Scand B 1982; 90: 13-19.

15. Stewart LD. Bacteroides fragilis in clinical infection. $(\mathrm{PhD}$ thesis) Queen's University of Belfast, Belfast, 1993.

16. Reid JH, Patrick S, Dermott E et al. Investigation of antigenic expression of Bacteroides fragilis by immunogold labelling and immunoblotting with a monoclonal antibody. FEMS Microbiol Lett 1985; 30: 289-293. 
17. Lutton DA, Variability of surface structure expression in Bacteroides fragilis. (PhD thesis) Queen's University of Belfast, Belfast, 1991.

18. Wilson KG. Investigation of the surface structures of Bacteroides fragilis using immunological methods. (MMedSc thesis) Queen's University of Belfast, Belfast, 1992.

19. Nikolich MP, Hong G, Shoemaker NB, Salyers AA. Evidence for natural horizontal transfer of tet $Q$ between bacteria that normally colonize humans and bacteria that normally colonize livestock. Appl Environ Microbiol 1994; 60: 3255-3260.

20. Kelly MJ. The quantitative and histological demonstration of pathogenic synergy between Escherichia coli and Bacteroides fragilis in guinea-pig wounds. J Med Microbiol 1978; 11 : 513-523.

21. Gossling J. Occurrence and pathogenicity of the Streptococcus milleri group. Rev Infect Dis 1988; 10: 257-285. 